\title{
Article \\ Chemical Characterization of Marrubium vulgare Volatiles from Serbia
}

\author{
Milica Aćimović ${ }^{1, *(1)}$, Stefan Ivanović ${ }^{2}\left(\mathbb{D}\right.$, Katarina Simić ${ }^{2}$, Lato Pezo ${ }^{3}$, , Tijana Zeremski ${ }^{1}$, Jelena Ovuka ${ }^{1}(\mathbb{D}$ \\ and Vladimir Sikora ${ }^{1}$
}

1 Institute of Field and Vegetable Crops Novi Sad, Maksima Gorkog 30, 21000 Novi Sad, Serbia; tijana.zeremski@ifvcns.ns.ac.rs (T.Z.); jelena.ovuka@ifvcns.ns.ac.rs (J.O.); vladimir.sikora@ifvcns.ns.ac.rs (V.S.)

2 Institute of Chemistry, Technology and Metallurgy, University of Belgrade, 11000 Belgrade, Serbia; stefan.ivanovic@ihtm.bg.ac.rs (S.I.); katarina.simic@ihtm.bg.ac.rs (K.S.)

3 Institute of General and Physical Chemistry, University of Belgrade, 11000 Belgrade, Serbia; latopezo@yahoo.co.uk

* Correspondence: milica.acimovic@ifvens.ns.ac.rs

Citation: Aćimović, M.; Ivanović, S.; Simić, K.; Pezo, L.; Zeremski, T.; Ovuka, J.; Sikora, V. Chemical Characterization of Marrubium vulgare Volatiles from Serbia. Plants 2021, 10, 600. https://doi.org/10.3390/ plants10030600

Academic Editor: Jésus Palá-Pául

Received: 28 February 2021

Accepted: 16 March 2021

Published: 23 March 2021

Publisher's Note: MDPI stays neutral with regard to jurisdictional claims in published maps and institutional affiliations.

Copyright: (c) 2021 by the authors. Licensee MDPI, Basel, Switzerland. This article is an open access article distributed under the terms and conditions of the Creative Commons Attribution (CC BY) license (https:// creativecommons.org/licenses/by/ $4.0 /)$.

\begin{abstract}
Marrubium vulgare is a cosmopolitan medicinal plant from the Lamiaceae family, which produces structurally highly diverse groups of secondary metabolites. A total of 160 compounds were determined in the volatiles from Serbia during two investigated years (2019 and 2020). The main components were $E$-caryophyllene, followed by germacrene D, $\alpha$-humulene and $\alpha$-copaene. All these compounds are from sesquiterpene hydrocarbons class which was dominant in both investigated years. This variation in volatiles composition could be a consequence of weather conditions, as in the case of other aromatic plants. According to the unrooted cluster tree with 37 samples of Marrubium sp. volatiles from literature and average values from this study, it could be said that there are several chemotypes: $E$-caryophyllene, $\beta$-bisabolene, $\alpha$-pinene, $\beta$-farnesene, $E$-caryophyllene + caryophyllene oxide chemotype, and diverse (unclassified) chemotypes. However, occurring polymorphism could be consequence of adaptation to grow in different environment, especially ecological conditions such as humidity, temperature and altitude, as well as hybridization strongly affected the chemotypes. In addition, this paper aimed to obtain validated models for prediction of retention indices (RIs) of compounds isolated from M. vulgare volatiles. A total of 160 experimentally obtained RIs of volatile compounds was used to build the prediction models. The coefficients of determination were 0.956 and 0.964 , demonstrating that these models could be used for predicting RIs, due to low prediction error and high $r^{2}$.
\end{abstract}

Keywords: horehound; GC-MS; retention indices; QSRR; boosted trees regression model

\section{Introduction}

Marrubium vulgare L., also known as white horehound, is a perennial species from the Lamiaceae family. It is indigenous to the region between the Mediterranean Sea and Central Asia; however, today it is found worldwide, apart from the coldest regions and high altitudes [1]. This plant is highly resistant to drought and due to this it grows well in semiarid areas [2]. Additionally, as it is a moderate salt-tolerant species this medicinal plant could be grown on saline soil [3]. The surface of $M$. vulgare vegetative and generative organs is densely clothed with glandular and nonglandular trichomes which accumulate secondary metabolites [4]. M. vulgare produces structurally highly diverse groups of secondary metabolites, thus represents a valuable source of bioactive compounds and preparations with health-promoting effects: antioxidant, hepatoprotective, antiproliferative, anti-inflammatory, antidiabetic, and antimicrobial [5]. The use of this herb in traditional medicine is recorded worldwide for ameliorating chronic cough and cold, numerous conditions related to skin, liver, gastric, heart, and immune system [6]. Generally, M. vulgare is poor in essential oil, and the major compounds are diverse [1,7-17]. 
This proves that there are various chemotypes of $M$. vulgare. The lack of information in this field is pointed out by Yabrir $[1,18]$. The studies about genus Marrubium are mainly focused on taxonomical, morphological, and genetic diversity [4,19-26].

The main aim of this investigation was to determine volatiles composition of $M$. vulgare grown in Serbia during two years and to compare its chemical composition with literature data not only of $M$. vulgare but with other species from this genus as well (M. anisodon, M. aschersonii, M. astracanicum, M. crassidens, $M$. deserti, $M$. duabense, M. parviflorum, $M$. peregrinum, M. persicum, M. propinquum, M. velutinum). Another goal was to establish the new quantitative structure retention relationship (QSRR) models for anticipating the retention indices (RIs) of certain compounds in M. vulgare volatiles obtained by GC-MS chromatography utilizing the genetic algorithm (GA) variable selection method and the boosted trees regression. Furthermore, we gather information about the volatile compounds of species from Marrubium genus in order to classify the chemotype of M. vulgare from this study according to unrooted cluster tree.

\section{Results}

The main components in $M$. vulgare volatiles were $E$-caryophyllene with $24.6 \%$ and $23.0 \%$, followed by germacrene $\mathrm{D}$ with $9.6 \%$ and $17.0 \%, \alpha$-humulene with $5.2 \%$ and $5.3 \%$ as well as $\alpha$-copaene with $3.3 \%$ and $6.1 \%$ in 2019 and 2020, respectively. All these compounds are from the sesquiterpene hydrocarbons class which was dominant in both years of the investigation, $52.0 \%$ in 2019 and $67.8 \%$ in 2020 . This variation in volatiles composition could be a consequence of weather conditions, as in case of other aromatic plants [27-33].

However, some of the components detected in $M$. vulgare volatiles during the twoyear research have not been detected yet in this species, while other components have not been detected in other species of this genus. ScienceDirect Elsevier, SpringerLink, PubMed, Scopus, Scifnder, Web of Science, Wiley Online, and Google Scholar databases were reviewed and scientific publications from 1990 until 2020 that deal with chemical composition of volatiles species from genus Marrubium were summarized and shown in Table 1.

Table 1. Chemical composition of Marrubium vulgare during two years (2019 and 2020).

\begin{tabular}{|c|c|c|c|c|c|c|c|c|}
\hline \multirow{2}{*}{ No } & \multirow{2}{*}{ Compound/Class } & \multirow{2}{*}{ Cycle } & \multirow{2}{*}{$\mathbf{R I}_{\text {pred. }}$} & \multicolumn{2}{|c|}{2019} & \multicolumn{2}{|c|}{2020} & \multirow{2}{*}{ Reference } \\
\hline & & & & $R I^{a}$ & $\%$ & $R I^{a}$ & $\%$ & \\
\hline 1 & 2E-Hexenal O & Train & 892.915 & - & - & 847 & 0.2 & $\begin{array}{l}\text { M. aschersonii [34], M. deserti [35], } \\
\text { M. peregrinum [36], M. vulgare [10,12,15,16,34] }\end{array}$ \\
\hline 2 & $\begin{array}{c}\text { Furan, } \\
\text { 2,5-diethyltetrahydro O }\end{array}$ & Validation & 853.684 & - & - & 897 & 0.1 & \\
\hline 3 & 1-Octen-3-ol O & Validation & 965.818 & 976 & 0.2 & 974 & 0.6 & $\begin{array}{l}\text { M. anisodon [37], M. astracanicum [38], } \\
\text { M. crassidens [39], M. deserti [35], M. duabense [40], } \\
\text { M. parviflorum [41,42], M. peregrinum [43,44], } \\
\text { M. persicum [45], M. propinquum [41], } \\
\text { M. velutinum [44], M. vulgare }[7,8,10,15]\end{array}$ \\
\hline 4 & 2-Pentyl furan $\mathrm{O}$ & Train & 1059.803 & - & - & 989 & 0.1 & \\
\hline 5 & 3-Octanol O & Test & 962.233 & - & - & 992 & 0.1 & $\begin{array}{l}\text { M. anisodon [37], M. astracanicum [46], } \\
\text { M. duabense [40], M. peregrinum }[36,44], \\
\text { M. velutinum [44] }\end{array}$ \\
\hline 6 & Linalool OMN & Train & 1106.041 & 1102 & 0.1 & 1098 & 0.1 & $\begin{array}{c}\text { M. aschersonii [34], M. astracanicum [46], } \\
\text { M. parviflorum }[41,42,47,48] \\
\text { M. peregrinum }[36,43,44], \text { M. persicum }[45], \\
\text { M. velutinum [44], M. vulgare }[8,10,12,17,34,36,47,49]\end{array}$ \\
\hline 7 & n-Nonanal O & Train & 1078.484 & - & - & 1102 & 0.1 & $\begin{array}{c}\text { M. aschersonii [34], M. deserti [35], M. duabense [40], } \\
\text { M. peregrinum [43,44], M. persicum [45], } \\
\text { M. velutinum [44], M. vulgare [34] }\end{array}$ \\
\hline 8 & E-Thujone OMN & Train & 1118.307 & - & - & 1114 & 0.1 & M. peregrinum [43], M. vulgare $[8,15]$ \\
\hline 9 & NI-1 & - & - & - & - & 1132 & 0.1 & - \\
\hline
\end{tabular}


Table 1. Cont.

\begin{tabular}{|c|c|c|c|c|c|c|c|c|}
\hline \multirow{2}{*}{ No } & \multirow{2}{*}{ Compound/Class } & \multirow{2}{*}{ Cycle } & \multirow{2}{*}{$\mathbf{R I}_{\text {pred. }}$} & \multicolumn{2}{|c|}{2019} & \multicolumn{2}{|c|}{2020} & \multirow{2}{*}{ Reference } \\
\hline & & & & $\mathbf{R I}^{\mathrm{a}}$ & $\%$ & $\mathbf{R I}^{\mathbf{a}}$ & $\%$ & \\
\hline 10 & Geijerene $\mathrm{O}$ & Train & 1192.301 & 1143 & 0.1 & 1139 & 0.6 & $\begin{array}{c}\text { M. incanum }[50,51], \text { M. parviflorum }[42,47] \\
\text { M. peregrinum }[43], \text { M. vulgare }[50]\end{array}$ \\
\hline 11 & 2E-Nonen-1-al O & Validation & 1097.602 & - & - & 1156 & 0.1 & \\
\hline 12 & $\beta$-Cyclocitral O & Train & 1216.889 & - & - & 1219 & 0.1 & M. peregrinum [44], M. velutinum [44], M. vulgare [10] \\
\hline 13 & Cogeijerene $\mathrm{O}$ & Train & 1203.235 & - & - & 1283 & 0.1 & \\
\hline 14 & Pregeijerene $\mathrm{O}$ & Train & 1149.857 & 1290 & 0.1 & 1287 & 0.2 & $\begin{array}{l}\text { M. astracanicum [38], M. crassidens [38], } \\
\text { M. parviflorum }[42,47], \text { M. peregrinum [43] }\end{array}$ \\
\hline 15 & Thymol AR & Test & 1209.017 & 1292 & 0.3 & - & - & M. deserti [52], M. vulgare $[7,8,10,15,50]$ \\
\hline 16 & 2-Undecanone $\mathrm{O}$ & Train & 1269.228 & 1295 & 0.1 & 1292 & Trace & M. vulgare [15] \\
\hline 17 & Carvacrol AR & Validation & 1179.072 & 1302 & 0.1 & - & - & $\begin{array}{c}\text { M. duabense [40], M. incanum [50], M. parviflorum [42], } \\
\text { M. peregrinum [43], M. vulgare }[7,8,10,49,50]\end{array}$ \\
\hline 18 & $\delta$-Elemene ST & Test & 1512.436 & - & - & 1336 & 0.1 & $\begin{array}{l}\text { M. anisodon [37], M. astracanicum [38], } \\
\text { M. crassidens [38], M. deserti }[35,40], \\
\text { M. duabense [40], M. incanum [50,51], } \\
\text { M. parviflorum [47], M. peregrinum [44], } \\
\text { M. persicum [53], M. thessalum }[54], \\
\text { M. velutinum [44], M. vulgare }[47,50]\end{array}$ \\
\hline 19 & $\alpha$-Cubebene ST & Train & 1491.202 & - & - & 1348 & 0.1 & $\begin{array}{l}\text { M. astracanicum [38], M. crassidens [39], } \\
\text { M. deserti }[35,40], \text { M. duabense [40], } \\
\text { M. parviflorum [47], M. peregrinum [44], } \\
\text { M. persicum [45], M. vulgare [8,47] }\end{array}$ \\
\hline 20 & Eugenol AR & Train & 1372.624 & - & - & 1357 & 0.4 & $\begin{array}{c}\text { M. aschersonii }[34], \text { M. peregrinum }[36,43,44] \\
\text { M. persicum }[53], \text { M. velutinum }[44], \\
\text { M. vulgare }[10,12,34,36,47]\end{array}$ \\
\hline 21 & $\alpha$-Copaene ST & Train & 1475.878 & 1377 & 3.3 & 1377 & 6.1 & $\begin{array}{l}\text { M. anisodon [37], M. aschersonii [34], } \\
\text { M. astracanicum [38], M. crassidens [38], } \\
\text { M. deserti [35], M. duabense [40], M. incanum [50,51], } \\
\text { M. parviflorum }[42,47,48], \text { M. peregrinum }[36,43,44] \text {, } \\
\text { M. persicum [53], M. thessalum }[54], \\
\text { M. velutinum }[44], \text { M. vulgare }[8-13,36,47,50]\end{array}$ \\
\hline 22 & $\beta$-Bourbonene ST & Train & 1487.976 & 1385 & 0.8 & 1384 & 1.2 & $\begin{array}{c}\text { M. anisodon }[37], \text { M. astracanicum }[38], \\
\text { M. crassidens [38], M. deserti }[35,52], \\
\text { M. incanum }[50,51], \text { M. parviflorum }[41,42,47,48] \text {, } \\
\text { M. peregrinum }[43,44], \text { M. persicum }[45,53], \\
\text { M. thessalum }[54], \text { M. velutinum }[44], \\
\text { M. vulgare }[9,10,13,50]\end{array}$ \\
\hline
\end{tabular}

\begin{tabular}{|c|c|c|c|c|c|c|c|c|}
\hline 23 & NI-2 & - & - & - & - & 1388 & 0.1 & - \\
\hline 24 & $\beta$-Cubebene ST & Test & 1475.610 & 1390 & 0.1 & 1389 & 0.2 & $\begin{array}{l}\text { M. aschersonii }[34], \text { M. deserti }[35], \\
\text { M. peregrinum }[43,44], \text { M. parviflorum }[42] \text {, } \\
\text { M. velutinum }[44], \text { M. vulgare }[12,13,34,47]\end{array}$ \\
\hline 25 & $\beta$-Elemene ST & Train & 1475.506 & 1392 & 0.4 & 1391 & 1.0 & $\begin{array}{c}\text { M. anisodon [37], M. astracanicum [38], } \\
\text { M. crassidens [38], M. deserti }[35,52], \\
\text { M. duabense [40], M. incanum }[50,51], \\
\text { M. parviflorum }[42,47], \text { M. peregrinum }[44], \\
\text { M. persicum }[53], \text { M. thessalum }[54], \\
\text { M. velutinum }[44], \text { M. vulgare }[47,50]\end{array}$ \\
\hline 26 & Z-Caryophyllene ST & Train & 1463.161 & 1407 & 0.1 & 1406 & 0.2 & \\
\hline 27 & $\alpha$-Z-Bergamotene ST & Train & 1428.215 & 1416 & 0.2 & - & - & \\
\hline 28 & E-Caryophyllene ST & Validation & 1463.161 & 1422 & 24.6 & 1423 & 23.0 & $\begin{array}{c}\text { M. anisodon }[37], \text { M. aschersonii }[34], \\
\text { M. astracanicum }[38,46], \text { M. crassidens }[38,39], \\
\text { M. deserti }[35,52], \text { M. duabense }[40], \\
\text { M. incanum }[50,51], \text { M. parviflorum }[41,42,47,48] \text {, } \\
\text { M. peregrinum }[36,43,44], \text { M. persicum }[45,53], \\
\text { M. propinquum }[41], \text { M. thessalum }[54], \\
\text { M. velutinum }[44], \\
\text { M. vulgare }[7-13,16,17,34,36,47,49,50]\end{array}$ \\
\hline 29 & $\beta$-Copaene ST & Test & 1459.623 & 1430 & 0.4 & 1430 & 1.3 & M. incanum [50], M. vulgare [50] \\
\hline
\end{tabular}


Table 1. Cont.

\begin{tabular}{|c|c|c|c|c|c|c|c|c|}
\hline \multirow{2}{*}{ No } & \multirow{2}{*}{ Compound/Class } & \multirow{2}{*}{ Cycle } & \multirow{2}{*}{$\mathrm{RI}_{\text {pred. }}$} & \multicolumn{2}{|c|}{2019} & \multicolumn{2}{|c|}{2020} & \multirow{2}{*}{ Reference } \\
\hline & & & & $\mathbf{R I}^{\mathrm{a}}$ & $\%$ & $\mathbf{R I}^{\mathrm{a}}$ & $\%$ & \\
\hline 30 & $\alpha$-E-Bergamotene ST & Train & 1428.215 & 1436 & 0.1 & 1435 & 0.1 & $\begin{array}{c}\text { M. anisodon [37], M. astracanicum [46], } \\
\text { M. crassidens [38], M. parviflorum }[42,47], \\
\text { M. peregrinum [44], M. velutinum [44], M. vulgare [47] }\end{array}$ \\
\hline 31 & NI-3 & - & - & 1445 & 0.2 & 1444 & 0.6 & - \\
\hline 32 & $\alpha$-Humulene ST & Validation & 1503.999 & 1454 & 5.2 & 1455 & 5.3 & $\begin{array}{l}\text { M. anisodon [37], M. aschersonii [34], } \\
\text { M. astracanicum [38,46], M. crassidens [38,39], } \\
\text { M. duabense [40], M. incanum }[50,51], \\
\text { M. parviflorum }[42,47], \text { M. peregrinum }[36,43], \\
\text { M. persicum [45], M. thessalum [54], M. velutinum [44], } \\
\text { M. vulgare }[8-10,12,13,15,34,36,47,50]\end{array}$ \\
\hline 33 & Sesquisabinene ST & Train & 1442.740 & - & - & 1457 & 0.9 & \\
\hline 34 & E- $\beta$-Farnesene ST & Test & 1431.419 & 1457 & 1.3 & - & - & $\begin{array}{l}\text { M. anisodon [37], M. aschersonii [34], M. crassidens [39], } \\
\text { M. parviflorum }[41,42,47], \text { M. peregrinum }[43,44], \\
\text { M. persicum }[45], \text { M. propinquum }[41], \\
\text { M. thessalum }[54], \text { M. velutinum }[44], \\
\text { M. vulgare }[8,10,12,16,17,34,36,47]\end{array}$ \\
\hline 35 & C16H34 A & Train & 1573.436 & 1462 & 1.5 & 1462 & 0.2 & \\
\hline 36 & NI-4 & - & - & - & Trace & - & 0.2 & - \\
\hline 37 & $\begin{array}{l}\text { Z-Muurola-4(14),5- } \\
\text { diene } \\
\text { ST }\end{array}$ & Train & 1482.433 & - & - & 1466 & 0.1 & \\
\hline 38 & NI-5 & - & - & 1469 & 0.1 & - & - & - \\
\hline 39 & NI-6 & - & - & 1472 & 0.1 & - & - & - \\
\hline 40 & $\begin{array}{l}\text { E-Cadina-1(6),4-diene } \\
\text { ST }\end{array}$ & Train & 1481.465 & - & - & 1475 & Trace & M. vulgare [15] \\
\hline 41 & $\gamma$-Muurolene ST & Test & 1450.203 & 1479 & 0.1 & - & - & $\begin{array}{l}\text { M. incanum }[50], \text { M. peregrinum }[43,44] \\
\text { M. parviflorum }[42], \text { M. velutinum }[44]\end{array}$ \\
\hline 42 & Germacrene D ST & Test & 1450.188 & 1483 & 9.6 & 1487 & 17.0 & $\begin{array}{c}\text { M. anisodon }[37], \text { M. aschersonii }[34], \\
\text { M. astracanicum }[38], \text { M. crassidens }[38,39], \\
\text { M. deserti }[35,52], \text { M. incanum }[50,51], \\
\text { M. parviflorum }[41,42,47,48] \\
\text { M. peregrinum }[36,43,44], \text { M. persicum }[45,53], \\
\text { M. propinquum }[41], \text { M. thessalum }[54], \\
\text { M. velutinum }[44], \\
\text { M. vulgare }[9-13,15-17,34,36,47,50]\end{array}$ \\
\hline 43 & $\mathrm{E}$ - $\beta$-Ionone $\mathrm{O}$ & Test & 1471.735 & 1486 & 0.4 & 1489 & Trace & $\begin{array}{c}\text { M. anisodon [37], M. aschersonii [34], } \\
\text { M. duabense [40], M. incanum }[51], \\
\text { M. parviflorum [42], M. peregrinum }[43,44] \\
\text { M. thessalum [54], M. vulgare }[12]\end{array}$ \\
\hline 44 & NI-7 & - & - & - & - & 1489 & 0.1 & - \\
\hline 45 & epi-Cubebol OST & Train & 1622.285 & - & - & 1495 & 0.2 & \\
\hline 46 & Viridiflorene ST & Validation & 1507.447 & 1497 & 0.1 & - & - & \\
\hline 47 & Bicyclogermacrene ST & Validation & 1493.697 & 1498 & 0.2 & 1498 & 0.2 & $\begin{array}{c}\text { M. astracanicum }[38], \text { M. crassidens }[38,39], \\
\text { M. deserti }[35,52], \text { M. duabense }[40], \\
\text { M. incanum }[50,51], \text { M. parviflorum }[41,42,47,48] \text {, } \\
\text { M. peregrinum }[36,43,44], \text { M. persicum }[45], \\
\text { M. propinquum }[41], \text { M. thessalum }[54], \\
\text { M. velutinum }[44], \text { M. vulgare }[10,11,17,50]\end{array}$ \\
\hline 48 & NI-8 & - & - & - & - & 1499 & 0.7 & - \\
\hline 49 & Pentadecane A & Test & 1486.884 & 1500 & 0.2 & 1500 & Trace & \\
\hline 50 & $\alpha$-Muurolene ST & Train & 1465.650 & 1501 & 0.1 & 1501 & 0.2 & $\begin{array}{c}\text { M. aschersonii [34], M. deserti }[35,52], M . \text { incanum [51], } \\
\text { M. peregrinum [43], M. velutinum }[44], \\
\text { M. vulgare }[12,13,34]\end{array}$ \\
\hline 51 & Germacrene A ST & Train & 1450.188 & 1508 & 0.1 & 1506 & 0.1 & M. incanum [50], M. parviflorum $[47,48]$ \\
\hline
\end{tabular}


Table 1. Cont.

\begin{tabular}{|c|c|c|c|c|c|c|c|c|}
\hline \multirow{2}{*}{ No } & \multirow{2}{*}{ Compound/Class } & \multirow{2}{*}{ Cycle } & \multirow{2}{*}{$\mathbf{R I}_{\text {pred. }}$} & \multicolumn{2}{|c|}{2019} & \multicolumn{2}{|c|}{2020} & \multirow{2}{*}{ Reference } \\
\hline & & & & $\mathbf{R I}^{\mathrm{a}}$ & $\%$ & $\mathbf{R I}^{\mathbf{a}}$ & $\%$ & \\
\hline 52 & $\beta$-Bisabolene ST & Validation & 1425.139 & 1511 & 0.2 & 1507 & 0.2 & $\begin{array}{c}\text { M. anisodon [37], M. aschersonii [34], } \\
\text { M. crassidens [38], M. parviflorum [47], } \\
\text { M. peregrinum [44], M. persicum }[45], \\
\text { M. propinquum [41], M. thessalum [54], } \\
\text { M. velutinum [44], M. vulgare [11-13,17,34,47,49] }\end{array}$ \\
\hline 53 & $\gamma$-Cadinene ST & Test & 1450.203 & 1513 & 0.2 & 1515 & 0.4 & $\begin{array}{c}\text { M. deserti }[52], \text { M. incanum }[50], \\
\text { M. parviflorum }[47,48], \text { M. peregrinum }[43,44], \\
\text { M. persicum }[53], \text { M. velutinum }[44], \\
\text { M. vulgare }[7,10,15,47]\end{array}$ \\
\hline 54 & $\delta$-Cadinene ST & Test & 1475.070 & 1523 & 4.7 & 1528 & 9.7 & $\begin{array}{c}\text { M. deserti [52], M. incanum }[50], \\
\text { M. parviflorum }[42,47], \text { M. peregrinum }[43,44], \\
\text { M. persicum [53], M. velutinum }[44], \\
\text { M. vulgare }[7,10,15,47]\end{array}$ \\
\hline 55 & E-Cadina-1,4-diene ST & Train & 1471.521 & 1533 & 0.1 & 1533 & 0.1 & M. vulgare [15] \\
\hline 56 & $\alpha$-Cadinene ST & Train & 1465.650 & - & - & 1537 & 0.1 & $\begin{array}{c}\text { M. peregrinum }[43,44], \text { M. velutinum }[44], \\
\text { M. vulgare }[47]\end{array}$ \\
\hline 57 & $\alpha$-Calacorene ST & Train & 1540.123 & - & - & 1543 & 0.1 & M. deserti [52], M. vulgare $[12,15]$ \\
\hline 58 & NI-9 & - & - & 1555 & 0.2 & 1552 & 0.2 & - \\
\hline 59 & E-Nerolidol OST & Validation & 1567.136 & 1561 & 3.5 & 1564 & 1.5 & $\begin{array}{c}\text { M. anisodon [37], M. deserti [52], M. parviflorum [42], } \\
\text { M. peregrinum }[43,44], \text { M. thessalum }[54], \\
\text { M. velutinum }[44], \text { M. vulgare }[9,36]\end{array}$ \\
\hline 60 & NI-10 & - & - & - & - & 1571 & 0.1 & - \\
\hline 61 & NI-11 & - & - & 1577 & 0.2 & 1575 & 0.9 & - \\
\hline 62 & NI-12 & - & - & - & - & 1582 & 0.3 & - \\
\hline 63 & $\begin{array}{l}\text { Caryophyllene } \\
\text { oxide OST }\end{array}$ & Test & 1636.612 & 1580 & 1.0 & 1583 & 1.8 & $\begin{array}{c}\text { M. anisodon }[37], \text { M. astracanicum }[46], \\
\text { M. crassidens }[38,39], \text { M. deserti }[52], \\
\text { M. duabense }[40], \text { M. incanum }[50,51], \\
\text { M. parviflorum }[41,42,47,48], \text { M. peregrinum }[36,43], \\
\text { M. persicum }[45,53], \text { M. propinquum }[41], \\
\text { M. thessalum }[54], \text { M. velutinum }[44], \\
\text { M. vulgare }[8-10,12,36,47,50]\end{array}$ \\
\hline 64 & NI-13 & - & - & - & - & 1587 & 0.1 & - \\
\hline 65 & Viridiflorol OST & Validation & 1573.436 & 1597 & 0.1 & - & - & $\begin{array}{l}\text { M. aschersonii [34], M. astracanicum [38], } \\
\text { M. crassidens [38], M. incanum [51], M. parviflorum [47], } \\
\text { M. peregrinum [43], M. vulgare [10,12,34,47] }\end{array}$ \\
\hline 66 & Hexadecane A & Train & 1594.576 & 1602 & 0.1 & - & - & M. duabense [40], M. velutinum [44] \\
\hline 67 & $\begin{array}{l}\text { Humulene epoxide } \\
\text { II OST }\end{array}$ & Train & 1626.959 & 1607 & 0.2 & 1607 & 0.2 & $\begin{array}{l}\text { M. anisodon [37], M. incanum [51], M. thessalum [54], } \\
\text { M. vulgare [10] }\end{array}$ \\
\hline 68 & $\begin{array}{l}\text { Muurola-4,10(14)- } \\
\text { dien-1- } \beta-\text { ol OST }\end{array}$ & Train & 1605.330 & - & - & 1627 & 0.3 & \\
\hline 69 & NI-14 & - & - & 1628 & 0.1 & - & - & - \\
\hline 70 & $\begin{array}{l}\text { 4,4-dimethyl- } \\
\text { Tetracyclo } \\
{[6.3 \cdot 2 \cdot 0(2,5) \cdot 0(1,8)]} \\
\text { tridecan-9-ol O }\end{array}$ & Validation & 1605.030 & - & - & 1631 & 0.2 & \\
\hline 71 & NI-15 & - & - & 1632 & 0.1 & - & - & - \\
\hline 72 & $\begin{array}{l}\text { Caryophylla-4(12), } \\
\text { 8(13)-dien-5- } \alpha \text {-ol OST }\end{array}$ & Train & 1605.030 & 1636 & 0.1 & 1635 & 0.3 & \\
\hline 73 & $\begin{array}{l}\text { epi- } \alpha \text {-Muurolol } \\
\text { (=tau-muurolol) OST }\end{array}$ & Test & 1605.030 & 1642 & 0.2 & 1641 & 0.6 & $\begin{array}{l}\text { M. astracanicum [38], M. deserti [35], } \\
\text { M. incanum [51], M. parviflorum [42], } \\
\text { M. peregrinum [44], M. velutinum [44] }\end{array}$ \\
\hline 74 & $\begin{array}{c}\alpha \text {-Muurolol } \\
\text { (=Torreyol) OST }\end{array}$ & Train & 1652.148 & - & - & 1645 & 0.1 & \\
\hline
\end{tabular}


Table 1. Cont.

\begin{tabular}{|c|c|c|c|c|c|c|c|c|}
\hline \multirow{2}{*}{ No } & \multirow{2}{*}{ Compound/Class } & \multirow{2}{*}{ Cycle } & \multirow{2}{*}{$\mathbf{R I}_{\text {pred. }}$} & \multicolumn{2}{|c|}{2019} & \multicolumn{2}{|c|}{2020} & \multirow{2}{*}{ Reference } \\
\hline & & & & $\mathbf{R I}^{\mathrm{a}}$ & $\%$ & $\mathbf{R I}^{\mathrm{a}}$ & $\%$ & \\
\hline 75 & $\alpha$-Cadinol OST & Train & 1682.934 & 1654 & 0.3 & 1654 & 0.9 & $\begin{array}{l}\text { M. crassidens [38], M. deserti }[35,52], \\
\text { M. incanum }[50,51], \text { M. parviflorum }[42], \\
\text { M. persicum [45], M. vulgare }[12,50]\end{array}$ \\
\hline 76 & NI-16 & - & - & 1658 & 0.2 & 1656 & 0.2 & - \\
\hline 77 & NI-17 & - & - & 1662 & 0.1 & 1662 & 0.1 & - \\
\hline 78 & $\begin{array}{l}\text { E-Calamenen- 10-ol } \\
\text { OST }\end{array}$ & Train & 1608.844 & - & - & 1669 & 0.1 & \\
\hline 79 & NI-18 & - & - & 1668 & 0.2 & - & - & - \\
\hline 80 & NI-19 & - & - & - & - & 1670 & 0.2 & - \\
\hline 81 & 8-Heptadecene O & Train & 1607.164 & - & - & 1673 & 0.2 & \\
\hline 82 & 1-Tetradecanol O & Train & 1702.771 & 1675 & 0.1 & - & - & \\
\hline 83 & $\begin{array}{c}\text { Germacra- } 4(15), 5 \\
\text { 10(14)-trien-1- } \alpha \text {-ol OST }\end{array}$ & Train & 1700.003 & 1682 & 0.1 & 1685 & 0.2 & \\
\hline 84 & Heptadecane A & Validation & 1726.886 & 1696 & 0.3 & 1696 & 0.2 & $\begin{array}{l}\text { M. anisodon [37], M. parviflorum [42,47], } \\
\text { M. vulgare [10,47] }\end{array}$ \\
\hline 85 & Pentadecanal O & Validation & 1581.928 & 1710 & 0.1 & 1711 & 0.1 & M. anisodon [37] \\
\hline 86 & Mint sulfide ST & Train & 1778.777 & 1733 & 0.1 & 1736 & 0.1 & \\
\hline 87 & NI-20 & - & - & 1734 & 0.1 & - & - & - \\
\hline 88 & NI-21 & - & - & 1742 & 0.1 & - & - & - \\
\hline 89 & NI-22 & - & - & 1743 & 0.4 & 1744 & 0.1 & - \\
\hline 90 & E-3-Octadecene O & Train & 1722.391 & - & - & 1777 & 0.1 & \\
\hline 91 & n-Pentadecanol O & Train & 1787.022 & 1778 & 0.1 & - & - & M. parviflorum [42] \\
\hline 92 & NI-23 & - & - & - & - & 1782 & 0.1 & - \\
\hline 93 & Octadecane A & Validation & 1950.093 & 1796 & 0.1 & - & - & $\begin{array}{c}\text { M. parviflorum [47], M. peregrinum [43], } \\
\text { M. vulgare [47] }\end{array}$ \\
\hline 94 & NI-24 & - & - & 1819 & 0.1 & - & - & - \\
\hline 95 & $\begin{array}{l}\text { 6,10,14-trimethyl- } \\
\text { 2-Pentadecanone O }\end{array}$ & Train & 1915.818 & 1844 & 4.8 & 1842 & 0.5 & $\begin{array}{c}\text { M. peregrinum [44], M. velutinum [44], } \\
\text { M. vulgare [10] }\end{array}$ \\
\hline 96 & NI-25 & - & - & 1849 & 0.1 & - & - & - \\
\hline 97 & NI-26 & - & - & 1853 & 0.2 & - & - & - \\
\hline 98 & NI-27 & - & - & 1888 & 0.1 & - & - & - \\
\hline 99 & NI-28 & - & - & 1891 & 0.8 & 1891 & 0.1 & - \\
\hline 100 & Nonadecane A & Test & 1869.346 & 1897 & 0.2 & 1897 & 0.2 & $\begin{array}{l}\text { M. duabense [40], M. parviflorum [47], } \\
\text { M. peregrinum [43], M. vulgare }[10,15,47]\end{array}$ \\
\hline 101 & NI-29 & - & - & 1904 & 0.1 & 1906 & Trace & - \\
\hline 102 & $\begin{array}{l}\text { 5E,9E-Farnesyl } \\
\text { acetone OST }\end{array}$ & Train & 1956.289 & 1916 & 0.3 & 1915 & Trace & M. thessalum [54], M. vulgare [15] \\
\hline 103 & NI-30 & - & - & 1918 & Trace & 1917 & Trace & - \\
\hline 104 & NI-31 & - & - & 1924 & 0.1 & - & - & - \\
\hline 105 & NI-32 & - & - & - & - & 1926 & Trace & - \\
\hline 106 & NI-33 & - & - & 1925 & 0.1 & - & - & - \\
\hline 107 & NI-34 & - & - & 1929 & 0.1 & - & - & - \\
\hline 108 & $\mathrm{NI}$ & & & 1938 & 0.1 & 1940 & Trace & \\
\hline 109 & Hexadecanoic acid $\mathrm{O}$ & Validation & 1995.491 & 1960 & 3.9 & - & - & $\begin{array}{c}\text { M. parviflorum [42], M. peregrinum [36], } \\
\text { M. vulgare }[36,47]\end{array}$ \\
\hline 110 & NI-35 & - & - & 1973 & 0.1 & 1974 & Trace & - \\
\hline 111 & Eicosane A & Train & 2034.560 & 1997 & 0.2 & 1994 & 0.1 & M. parviflorum [48] \\
\hline 112 & NI-36 & - & - & 2001 & 0.1 & - & - & - \\
\hline
\end{tabular}


Table 1. Cont.

\begin{tabular}{|c|c|c|c|c|c|c|c|c|}
\hline \multirow{2}{*}{ No } & \multirow{2}{*}{ Compound/Class } & \multirow{2}{*}{ Cycle } & \multirow{2}{*}{$\mathbf{R I}_{\text {pred. }}$} & \multicolumn{2}{|c|}{2019} & \multicolumn{2}{|c|}{2020} & \multirow{2}{*}{ Reference } \\
\hline & & & & $\mathrm{RI}^{\mathrm{a}}$ & $\%$ & $R I^{a}$ & $\%$ & \\
\hline 113 & $\begin{array}{l}\text { E,E-Geranyl } \\
\text { linalool OD }\end{array}$ & Train & 2028.645 & 2027 & 1.6 & - & - & $\begin{array}{c}\text { M. aschersonii [34], M. parviflorum [42], } \\
\text { M. vulgare }[12,34]\end{array}$ \\
\hline 114 & $\begin{array}{c}\text { 3,7,11,15-tetramethyl- } \\
\text { (E,E)-1,6,10,14- } \\
\text { Hexadecatetraen- } \\
\text { 3-ol OD }\end{array}$ & & & - & - & 2028 & 0.9 & \\
\hline 115 & Manool OD & Train & 2064.196 & 2057 & 0.3 & - & - & \\
\hline 116 & NI-37 & - & - & 2061 & 0.1 & - & - & - \\
\hline 117 & NI-38 & - & - & 2067 & 0.1 & - & - & - \\
\hline 118 & NI-39 & - & - & 2084 & 0.1 & - & - & - \\
\hline 119 & NI-40 & - & - & 2096 & 0.1 & - & - & - \\
\hline 120 & Heneicosane A & Train & 2120.284 & 2101 & 1.6 & 2100 & 1.3 & $\begin{array}{l}\text { M. parviflorum [42,47], M. peregrinum [43], } \\
\text { M. propinquum [41], M. vulgare [10] }\end{array}$ \\
\hline 121 & NI-41 & - & - & 2108 & 0.3 & 2105 & 0.2 & - \\
\hline 122 & NI-42 & - & - & 2112 & 0.2 & 2110 & 0.3 & - \\
\hline 123 & Phytol OD & Test & 2124.818 & 2116 & 1.4 & 2113 & 0.4 & $\begin{array}{l}\text { M. anisodon [37], M. incanum [51], } \\
\text { M. parviflorum [41,42], M. peregrinum [36], } \\
\text { M. propinquum [41], M. vulgare }[10,15]\end{array}$ \\
\hline 124 & NI-43 & - & - & 2131 & 0.2 & - & - & - \\
\hline 125 & NI-44 & - & - & 2143 & 0.2 & 2143 & 0.1 & - \\
\hline 126 & NI-45 & - & - & 2147 & 0.2 & - & - & - \\
\hline 127 & NI-46 & - & - & 2164 & 0.2 & 2160 & 0.2 & - \\
\hline 128 & NI-47 & - & - & 2167 & 0.1 & 2172 & 0.3 & - \\
\hline 129 & NI-48 & - & - & 2175 & 0.6 & 2176 & 0.4 & - \\
\hline 130 & NI-49 & - & - & 2181 & 0.9 & 2179 & 0.2 & - \\
\hline 131 & NI-50 & - & - & 2183 & 0.4 & - & - & - \\
\hline 132 & NI-51 & - & - & 2198 & 2.4 & 2195 & 2.4 & - \\
\hline 133 & Docosane A & Validation & 2194.421 & 2205 & 0.9 & 2198 & 0.6 & M. crassidens [39], M. parviflorum [47] \\
\hline 134 & NI-52 & - & - & - & - & 2201 & 0.1 & - \\
\hline 135 & NI-53 & - & - & - & - & 2209 & 0.3 & - \\
\hline 136 & NI-54 & - & - & 2215 & 0.3 & - & - & - \\
\hline 137 & NI-55 & - & - & 2225 & 0.3 & 2221 & 0.1 & - \\
\hline 138 & NI-56 & - & - & 2246 & 0.3 & - & - & - \\
\hline 139 & NI-57 & - & - & 2258 & 0.2 & 2253 & 0.3 & - \\
\hline 140 & NI-58 & - & - & 2270 & 0.1 & 2265 & 0.1 & - \\
\hline 141 & NI-59 & - & - & 2277 & 0.2 & 2274 & 0.2 & - \\
\hline 142 & NI-60 & - & - & 2293 & 3.8 & 2288 & 1.7 & - \\
\hline 143 & Tricontane A & Train & 2381.642 & 2305 & 3.6 & 2302 & 2.6 & \\
\hline 144 & NI-61 & - & - & 2309 & 0.2 & 2305 & 0.2 & - \\
\hline 145 & NI-62 & - & - & 2344 & 0.2 & 2341 & 0.3 & - \\
\hline 146 & NI-63 & - & - & 2380 & 0.1 & 2377 & 0.1 & - \\
\hline 147 & NI-64 & - & - & 2383 & 0.1 & 2382 & 0.1 & - \\
\hline 148 & Tetracosane A & Train & 2493.491 & 2401 & 0.3 & 2395 & 0.2 & $\begin{array}{c}\text { M. deserti [52], M. parviflorum }[41,42,47], \\
\text { M. propinquum }[41]\end{array}$ \\
\hline 149 & NI-65 & - & - & 2454 & 0.4 & 2447 & 0.2 & - \\
\hline 150 & NI-66 & - & - & 2488 & 0.2 & 2483 & 0.2 & - \\
\hline 151 & Pentacosane A & Test & 2510.087 & 2503 & 0.8 & 2497 & 0.6 & M. anisodon [37], M. parviflorum $[42,47]$ \\
\hline
\end{tabular}


Table 1. Cont.

\begin{tabular}{|c|c|c|c|c|c|c|c|c|}
\hline \multirow{2}{*}{ No } & \multirow{2}{*}{ Compound/Class } & \multirow{2}{*}{ Cycle } & \multirow{2}{*}{$\mathbf{R I}_{\text {pred. }}$} & \multicolumn{2}{|c|}{2019} & \multicolumn{2}{|c|}{2020} & \multirow{2}{*}{ Reference } \\
\hline & & & & $\mathbf{R I}^{\mathrm{a}}$ & $\%$ & $\mathbf{R I}^{\mathrm{a}}$ & $\%$ & \\
\hline 152 & Heptacosane A & Train & 2730.537 & 2702 & 0.6 & 2696 & 0.5 & $\begin{array}{l}\text { M. anisodon [37], M. aschersonii [34], } \\
\text { M. incanum [51], M. parviflorum [42], M. vulgare [34] }\end{array}$ \\
\hline 153 & NI-67 & - & - & - & - & 2766 & 0.1 & - \\
\hline 154 & Octacosane A & & & 2801 & 0.1 & 2791 & Trace & $\begin{array}{l}\text { M. crassidens [39], M. parviflorum [42], } \\
\text { M. persicum [45] }\end{array}$ \\
\hline 155 & Squalene $\mathrm{T}$ & Train & 2870.673 & 2835 & 0.1 & 2823 & 0.1 & \\
\hline 156 & NI-68 & - & - & 2868 & 0.1 & 2855 & 0.1 & - \\
\hline 157 & Nonacosane A & Test & 2930.732 & 2905 & 0.7 & 2892 & 0.6 & M. anisodon [37], M. crassidens [39], M. persicum [45] \\
\hline 158 & Untriacontane A & Validation & 3150.673 & 3105 & 0.4 & 3095 & 0.3 & \\
\hline 159 & NI-69 & - & - & - & - & 3212 & 0.1 & - \\
\hline 160 & Tritriacontane A & Train & 3319.753 & 3300 & 0.1 & 3301 & Trace & \\
\hline & $\begin{array}{c}\text { Oxygenated } \\
\text { monoterpenes OMN }\end{array}$ & & & & 0.1 & & 0.2 & \\
\hline & $\begin{array}{l}\text { Sesquiterpene } \\
\text { hydrocarbons ST }\end{array}$ & & & & 52.0 & & 67.8 & \\
\hline & $\begin{array}{l}\text { Oxygenated } \\
\text { sesquiterpenes OST }\end{array}$ & & & & 5.8 & & 6.2 & \\
\hline & $\begin{array}{l}\text { Oxygenated } \\
\text { diterpenes OD }\end{array}$ & & & & 3.3 & & 1.3 & \\
\hline & Triterpene $\mathrm{T}$ & & & & 0.1 & & 0.1 & \\
\hline & Aromatics AR & & & & 0.4 & & 0.4 & \\
\hline & Alkanes A & & & & 11.7 & & 7.4 & \\
\hline & Other O & & & & 9.9 & & 3.4 & \\
\hline & NI & & & & 16.7 & & 12.5 & \\
\hline & Total & & & & 100 & & 99.3 & \\
\hline
\end{tabular}

$\mathrm{RI}_{\text {pred. }}$ - BRT calculated retention index; $\mathrm{RI}^{\mathrm{a}}$ —retention index experimentally obtained on a HP-5MS column; Other-aliphatic hydrocarbons, aliphatic aldehydes and alcohols, aliphatic acids, their esters and aldehydes, aromatic esters with aliphatic acids, alkyl-aromatic alcohols, or aryl esters of aromatic acids; NI—not identified compound.

The predicted RIs are shown in Table 1, and confirm the good quality of the constructed BRT model by showing the relationship between the predicted and experimental RI values. Graphical comparison between experimentally obtained RIs of M. vulgare volatiles composition $\left(\mathrm{RI}^{\mathrm{a}}\right)$, the retention time indices found in NIST database $\left(\mathrm{RI}^{\mathrm{b}}\right)$ and the retention time indices predicted by the two BRT models $\left(\mathrm{RI}_{\text {pred. }}\right.$ ) are presented in Figure 1.

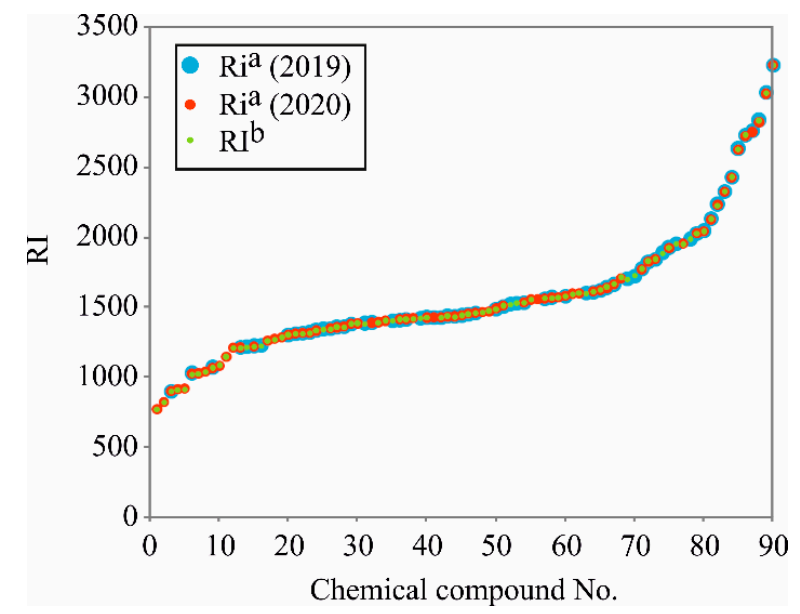

Figure 1. Retention indices (RIs) of the M. vulgare volatiles composition, from experimentally obtained GC-MS data on a HP-5MS column $\left(\mathrm{RI}^{\mathrm{a}}\right)$ and NIST database $\left(\mathrm{RI}^{\mathrm{b}}\right)$. 
In order to calculate the molecular descriptors, the PaDel-descriptor was used in this investigation. Due to a great amount of data that was obtained, it was required to select the most important set of descriptors to build the adequate model which would be able to predict the RIs [55]. The factor analysis was done before the GA calculation, and only ca. 320 uncorrelated descriptors remained in the GA calculation [56,57]. The seven most significant molecular descriptors chosen by GA are as follows: four 2D autocorrelation descriptors (AATSC4e, AATSC2p, GATS6v and MATS5v), two Barysz matrix descriptors (VR1_Dzs and SM1_Dzv) and Vertex adjacency information (magnitude) descriptor (VAdjMat).

The predicted RIs and molecular descriptors are presented in Table 1. Seven molecular descriptors were utilized for predictions of RIs in the two BRT models. The predicted RIs are presented in Figure 2, and visually confirm the adequate prediction capabilities of the constructed BRT by showing the relationship between the predicted and experimental retention values.
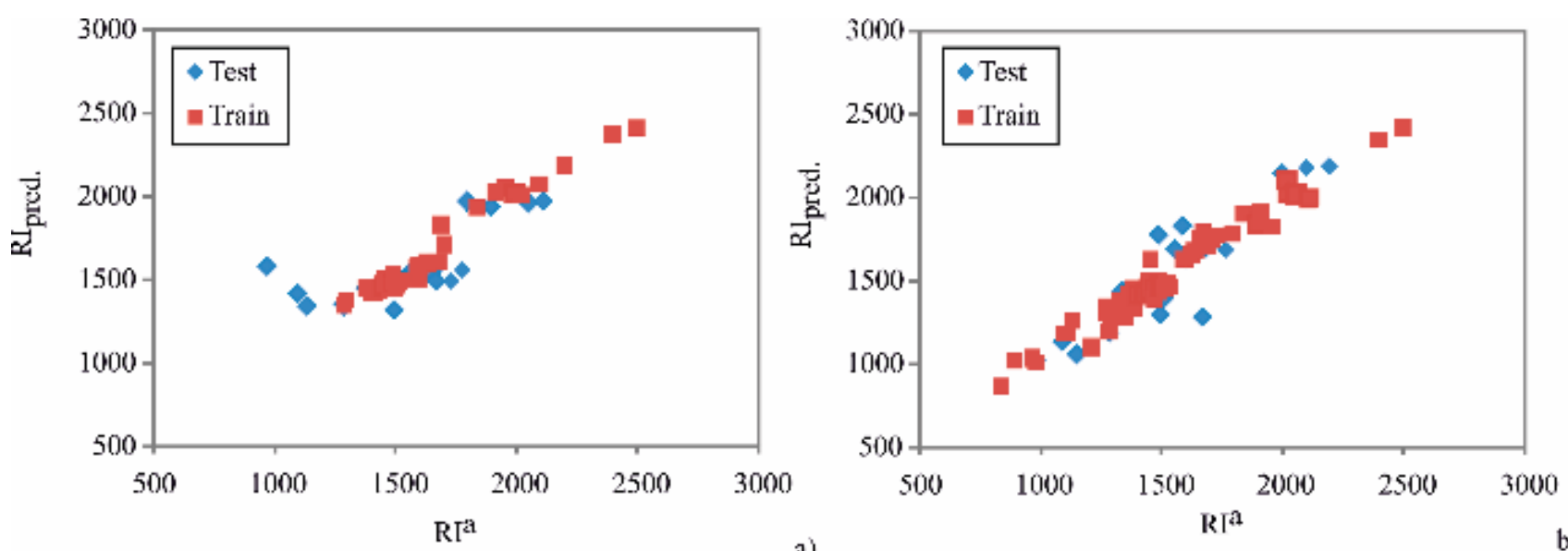

a)

b)

Figure 2. Comparison of experimentally obtained RIs on a HP-5MS column (RIa) with BRT pre-dicted values (RIpred.) in 2019 (a) and 2020 (b).

Separation of compounds in GC-MS and their RIs is linked to their affinity towards mobile and stationary phases. Affinity and solubility of separated molecules directly depend on their chemical structure and physico-chemical properties, which could be expressed by molecular descriptors. According to Pearson's correlation coefficients, there was a rather poor correlation between all 3D autocorrelation descriptors (Table 2). Therefore, utilized molecular descriptors were appropriate to predict RIs of compounds in M. vulgare by the two multivariate BRT models [58].

Table 2. The correlation coefficient matrix for the selected descriptors by GA.

\begin{tabular}{ccccccc}
\hline & AATSC2p & MATS5v & GATS6v & SM1_Dzv & VR1_Dzs & VAdjMat \\
\hline AATSC4e & 0.031 & -0.138 & -0.135 & 0.030 & 0.205 & 0.224 \\
AATSC2p & & -0.265 & -0.131 & 0.036 & -0.231 & -0.010 \\
MATS5v & & & 0.212 & -0.008 & 0.066 & 0.109 \\
GATS6v & & & & 0.072 & 0.131 & 0.214 \\
SM1 Dzv & & & & & 0.058 & 0.084 \\
VR1 Dzs & & & & & & 2.339 \\
\hline
\end{tabular}

Detailed explanations about the descriptors were found in the Handbook of Molecular Descriptors [59]. These descriptors encode different aspects of the molecular structure and were applied to develop the QSRR model. According to Pearson's correlation, there was a rather poor correlation between all molecular descriptors. Hence, utilized descriptors were appropriate to predict RIs of compounds isolated from $M$. vulgare volatiles by the two multivariate BRT models. The calibration and predictive capability of a QSRR model 
should be tested through model validation. The most widely used squared correlation coefficient $\left(r^{2}\right)$ can provide a reliable indication of the fit of the model, thus, it was employed to validate the calibration capability of a QSRR model.

In order to explore the nonlinear relationship between RIs and the descriptors selected by GA, BRT technique was used to build the two predictive models. Two BRT models were constructed to predict the retention time of compounds isolated from $M$. vulgare volatiles, respectively. The coefficients of determination were 0.956 and 0.964 , respectively, indicating that these models could be used for prediction of RIs, due to low prediction error and high $r^{2}$. The tests of the two BRT models fit (2019 and 2020) are shown in Table 3, with the higher $r^{2}$ values and lower $\chi^{2}$, MBE, RMSE, and MPE values showing the better fit to the experimental results $[60,61]$.

Table 3. The "goodness of fit" tests for the developed BRT model.

\begin{tabular}{cccccc}
\hline Boosted Tree Model & $\chi^{2}$ & RMSE & MBE & MPE & $r^{2}$ \\
\hline 2019 & 4455.272 & 66.160 & -13.063 & 3.285 & 0.956 \\
2020 & 3975.751 & 62.581 & -7.698 & 3.241 & 0.964 \\
\hline
\end{tabular}

$\chi^{2}$-reduced chi-square, MBE-mean bias error, RMSE-root mean square error, MPE-mean percentage error.

Obtained results reveal the reliability of the BRT models for predicting the RIs of compounds in M. vulgare volatiles obtained by GC-MS analysis. The influence of the seven most important molecular descriptors, identified by using genetic algorithm on the RIs was studied in this section. According to the Figure 3, VAdjMat was the most important molecular descriptor for chemical compounds' RIs calculation in M. vulgare, during 2019, while VR1 Dzs was the most important variable during 2020.
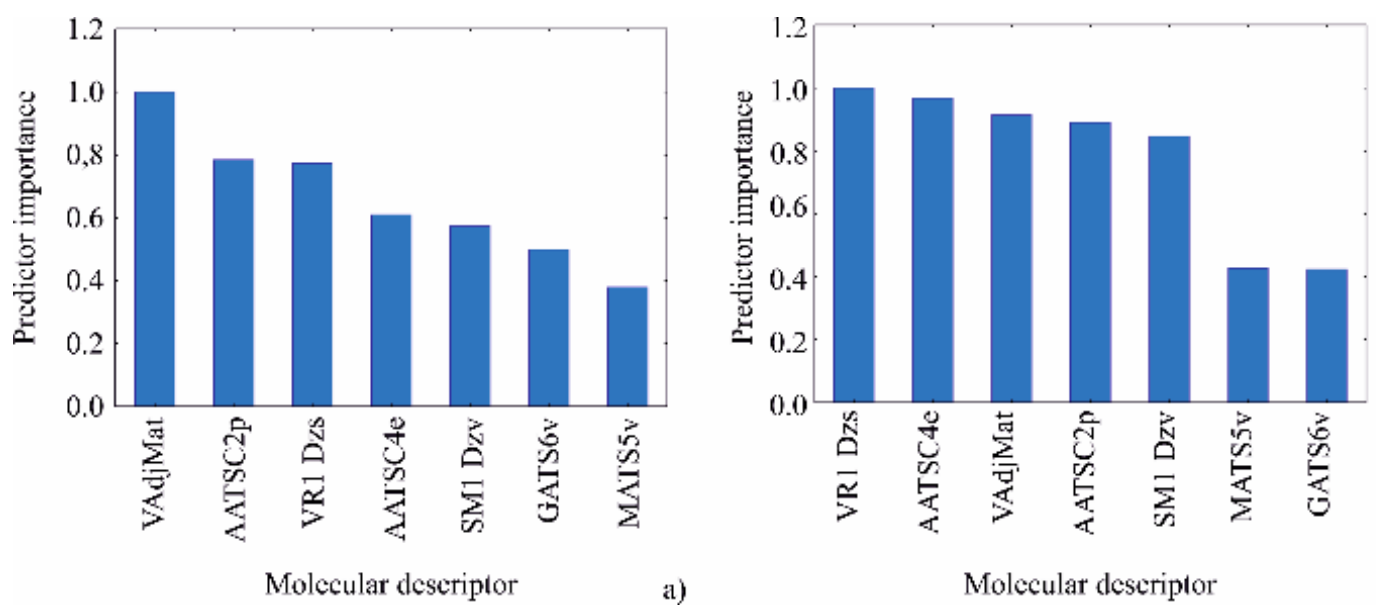

Figure 3. Predictor importance of the molecular descriptors on RI in 2019 (a) and 2020 (b).

\section{Discussion}

According to the cluster analysis (unrooted cluster tree) with 37 samples of Marrubium $\mathrm{sp}$. volatiles from literature and average values from this study (Figure 4), it could be said that there are several chemotypes, but only E-caryophyllene chemotype $[9,12,36,38,47,50,51]$ is clearly segregated. However, these are samples of $M$. vulgare, $M$. incanum, $M$. parviflorum, M. peregrinum, and M. crassidens grown in Serbia, Poland, Slovakia, Egypt, and Iran. This indicated that genus Marrubiumis very diverse in the case of volatiles composition. 


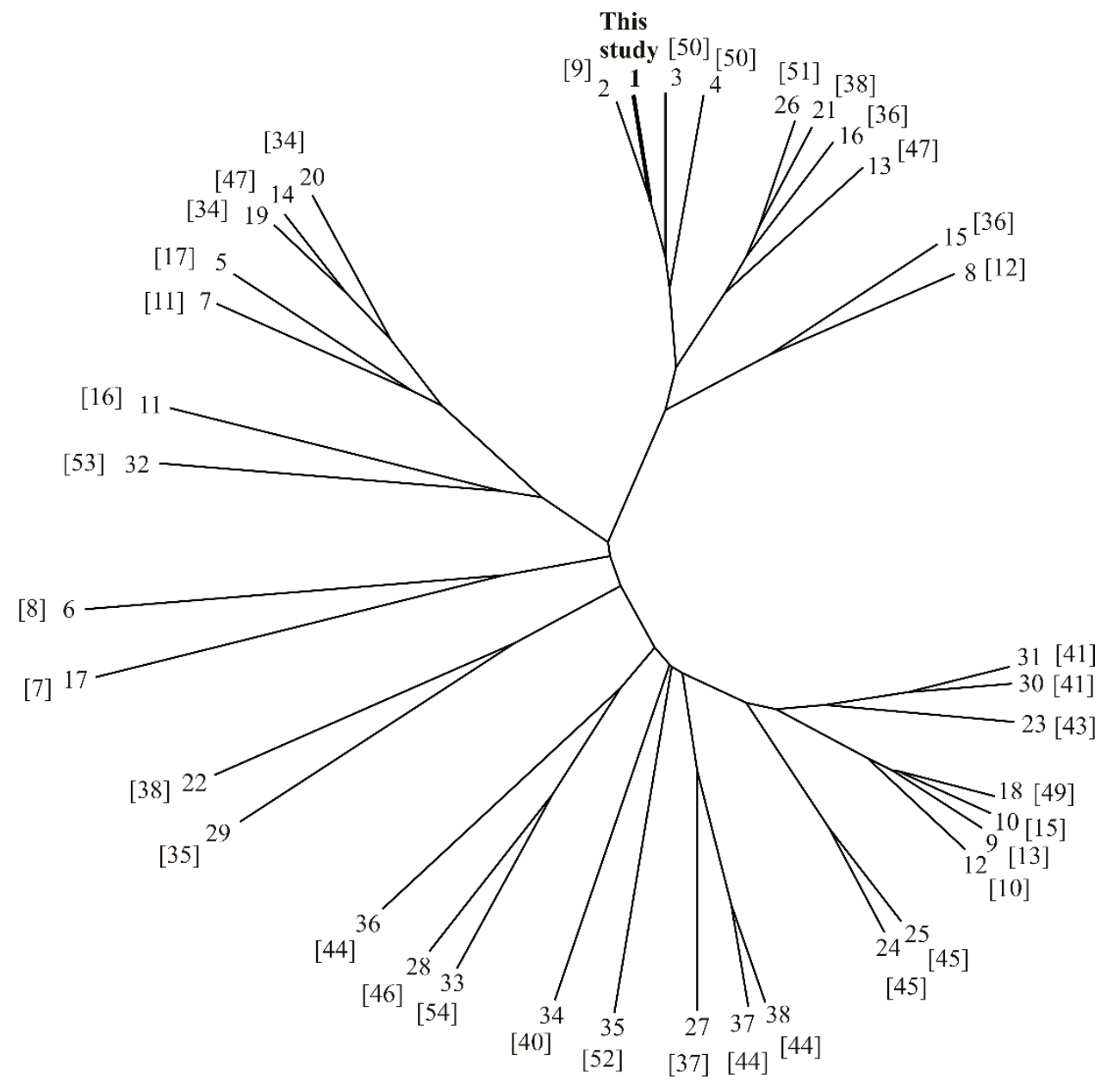

Figure 4. Unrooted cluster tree for different Marrubium samples.

In addition, $E$-caryophyllene is a compound which is occurring in all samples (except M. vulgare from Eastern Algeria [15]), but in E-caryophyllene chemotype its content ranges between $15.6 \%$ and $45.8 \%$. Other chemotypes can be classified as $\beta$-bisabolene (13.1-28.3\%) [11,17,34,47], $\alpha$-pinene (21.5-28.9\%) [16,53], $\beta$-farnesene (20.2-24.2\%) [37,44], E-caryophyllene + caryophyllene oxide chemotype [44,46,54], and diverse (unclassified) chemotypes $[7,8,10,13,15,35,38,40,41,43,45,49,52]$.

Occurring polymorphism could be a consequence of adaptation to grow in different environments [19,62], especially ecological conditions such as humidity, temperature and altitude [22] as well as hybridization [20] strongly affected the chemotypes, as well as biotic and abiotic stresses (including temperature, light, water, salt, and oxidative stresses) [63].

Detected compounds in M. vulgare volatiles obtained by GC-MS analysis were used for QSRR analysis. The following seven molecular descriptors that characterize the RIs of obtained compounds were suggested by the genetic algorithm. Selected molecular descriptors were not autocorrelated which was suggested by a correlation coefficient matrix; thus, descriptors were suitable for QSRR analysis. These descriptors were utilized as inputs for the boosted trees regression models, for estimating the RIs using a set of GCMS data from a series of 160 compounds found in M. vulgare volatiles. Statistical models that quantify the relation between the structure of molecules and their chromatographic RIs were represented by the quantitative structure retention relationship (QSRR) model $[58,64]$. Numerous publications related to the QSRR analysis in plants from Lamiaceae family could be found in the literature: Thymus vulgaris [65], T. serpyllum [66], Satureja kitaibelii [55], Salvia officinalis [67], as well as Stachys sp. [68]. The connection between the molecular 
descriptors and the retention time can be established by artificial neural network, machine learning algorithms [69-73] or by boosted trees regression (BRT) [74].

\section{Materials and Methods}

\subsection{Plant Material}

M. vulgare was grown in the Institute of Field and Vegetable Crops (IFVCNS) collection garden of medicinal and aromatic plants in Bački Petrovac $\left(45^{\circ} 21^{\prime} \mathrm{N} ; 19^{\circ} 35^{\prime} \mathrm{E}\right)$, confirmed by Milica Rat, PhD, and deposited at the Herbarium BUNS (Department of Biology and Ecology, Faculty of Sciences, University of Novi Sad) under the voucher number 2-1409. After seed maturation (August 2018), it was collected and sown in field conditions in September 2018 and 2019. The experimental plot was $10 \mathrm{~m}$ long and $5 \mathrm{~m}$ wide, with a $70 \mathrm{~cm}$ spacing between rows. From the seven rows, only three central rows were used for collecting plant material to avoid edge effects (one row one sample).

\subsection{Volatiles Isolation and Analysis}

Flowering aerial parts of M. vulgare (Marrubii herba) were collected during July 2019 and 2020, dried in a solar dryer at a temperature of $40^{\circ}$ with air circulation. After drying, plant material was fragmented, and volatiles was extracted by Clevenger apparatus. Taking in account that $M$. vulgare produces trace amounts of essential oil, it was trapped in nhexane. This process was performed in tree repetition for both years, as well as analysis of chemical composition.

GC-MS analysis was carried out using an Agilent 7890A apparatus equipped with a 5975 C MSD, FID and a nonpolar HP-5MS fused-silica capillary column $(30 \mathrm{~m} \times 0.25 \mathrm{~mm}$, film thickness $0.25 \mu \mathrm{m}$ ). The carrier gas was helium, and its inlet pressure was $19.6 \mathrm{psi}$ and linear velocity of $1 \mathrm{~mL} / \mathrm{min}$ at $210{ }^{\circ} \mathrm{C}$. The injector temperature was $250{ }^{\circ} \mathrm{C}$, injection volume was $1 \mu \mathrm{L}$, split ratio, 10:1. Mass detection was carried out under source temperature conditions of $230^{\circ} \mathrm{C}$ and interface temperature of $315^{\circ} \mathrm{C}$. The EI mode was set at electron energy, $70 \mathrm{eV}$ with mass scan range of 40-600 amu. Temperature was programmed from 60 to $300{ }^{\circ} \mathrm{C}$ at a rate of $3{ }^{\circ} \mathrm{C} / \mathrm{min}$. The components were identified based on their linear retention index relative to $\mathrm{C} 8$-C $32 \mathrm{n}$-alkanes, compared with data reported in the literature (Adams4 and NIST11 databases). The relative percentage of the oil constituents was expressed as percentages by FID peak area normalization.

\subsection{QSRR Analysis}

PaDel-descriptor software was used to calculate specified molecular descriptors [75], as described in our previous investigation [66]. Factor analysis and genetic algorithm (GA) were used to determine the most important descriptors [76,77]. The relationship between the chosen descriptors was examined and collinear descriptors were excluded. Statistica 10 software was used for the statistical investigation of the data [78].

\subsection{BRT Model}

In order to relate and to predict categorical or continuous dependent variables the BRT model could be used [79,80], as it does not require transformation or outliers [81]. The BRT method calculation is connected to the boosting methods enforced to regression trees [82]. The main idea is to calculate a set of simple trees, where each successive tree is built for the prediction residuals of the preceding tree [83]. This method builds binary trees such as partition the data into two samples at each split node [78].

The decision trees are combined through a cross-validation or "boosting" procedure in order to acquire the single computational model [84]. BRT modeling consists of the following steps: (a) an initial regression tree is defined according to a minimum loss function; (b) the other trees are engaged in the iterative process in which several new regression trees were developed and selected to the subsequent according to the StatSoft Statistica's recommendation - the least square error (LSE); (c) step (b) is repeated until a stopping criterion is reached (for instance, the value of LSE). 
In this study, several regularization parameters were set in order to optimize the fit between experimental results and computing model: the number of trees (between 100 and 1000), learning rate (between 0.0005 and 0.1 ), random test data proportion (0.1-0.9) and subsample proportion (0.1-0.9). According to Statistica's manual, prior to computation, a subsample of data is created, according to random test data proportion of the cases, and these data are treated as test samples used to evaluate the appropriate fit of the model. The remaining set of data is used for the analyses via stochastic gradient boosting (for the selection of samples for consecutive boosting steps).

\subsection{Cluster Analysis}

The cluster analysis (CA) was used to evaluate intra- and interpopulation variability and differentiation of volatile constituents of Marrubium samples collected in different locations and/or taken from literature reports. The phylogenetic tree diagram for Marrubium samples was calculated and plotted using R software 4.0.3 (64-bit version). The R package "ape" (Analysis of Phylogentics and Evolution) was used for calculation, applied as a graphical tool to represent the arrangements of similar volatiles concentration (evaluated in the cluster analysis). The obtained experimental results were collected in the matrix, after which the hierarchical cluster analysis was performed. The distance matrix was determined using the Euclidean method, while the cluster analysis was performed using the "complete" method.

\section{Conclusions}

The main components in M. vulgare volatiles were E-caryophyllene (24.6\% and 23.0\%), followed by germacrene D (9.6\% and $17.0 \%), \alpha$-humulene (5.2\% and $5.3 \%)$ and $\alpha$-copaene $(3.3 \%$ and $6.1 \%)$ in 2019 and 2020, respectively. All these compounds are from sesquiterpene hydrocarbons class, which was dominant in both years of the investigation, 52.0\% in 2019 and $67.8 \%$ in 2020 .

The results demonstrated that the boosted trees regression models were adequate in predicting the RIs of the compounds in M. vulgare volatiles obtained by GC-MS analysis on a HP-5MS column. The coefficients of determination were 0.956 and 0.964 (for compounds found in M. vulgare volatiles, during the years 2019 and 2020, respectively), which is a good indication that these models could be used as a fast mathematical tool for prediction of RIs, due to low prediction error and moderately high $r^{2}$. Suitable models with high statistical quality and low prediction errors were derived, and it could be further used for estimation of RIs of newly detected compounds.

According to the unrooted cluster tree with 37 samples of Marrubium sp. volatiles from literature and average values from this study, it could be said that there are several chemotypes: $E$-caryophyllene, $\beta$-bisabolene, $\alpha$-pinene, $\beta$-farnesene, $E$-caryophyllene + caryophyllene oxide chemotype, and diverse (unclassified) chemotypes. However, occurring polymorphism could be a consequence of adaptation to grow in different environments, especially ecological conditions such as humidity, temperature and altitude, as well as hybridization which strongly affected the chemotypes. Further research on M. vulgare chemotypes needs to be focused on genetic markers, because evaluation of genetic diversity has key importance in improving the quality of raw material used for industrial purposes.

Author Contributions: Conceptualization, M.A. and J.O.; methodology, S.I. and K.S.; software, L.P.; validation, S.I., K.S. and T.Z.; formal analysis, L.P.; investigation, M.A.; resources, M.A. and V.S.; data curation, L.P.; writing—original draft preparation, M.A.; writing—review and editing, T.Z. and V.S.; visualization, L.P.; supervision, J.O.; project administration, T.Z.; funding acquisition, M.A., T.Z., J.O. and V.S. All authors have read and agreed to the published version of the manuscript.

Funding: This research was supported by the Ministry of Education, Science and Technological Development of the Republic of Serbia, grant number: 451-03-9/2021-14/200032.

Data Availability Statement: Not applicable. 
Acknowledgments: We thank Vele Tešević, Marina Todosijević, Jovana Stanković Jeremić, Jovana Ljujić, and Mirjana Cvetković for participating in this research.

Conflicts of Interest: The authors declare no conflict of interest.

\section{References}

1. Yabrir, B. Essential oil of Marrubium vulgare: Chemical composition and biological activities. A review. Nat. Prod. Sci. 2019, 25, 81-91. [CrossRef]

2. Lippai, A.; Smith, P.A.; Price, T.V.; Weiss, J.; Lloyd, C.J. Effects of temperature and water potential on germination of horehound (Marrubium vulgare) seeds from two Australian Localities. Weed Sci. 1996, 44, 91-99. [CrossRef]

3. Nedjimi, B.; Souissi, Z.E.; Guit, B.; Daoud, Y. Differential effects of soluble salts on seed germination of Marrubium vulgare L. J. Appl. Res. Med. Aromat. Plants 2020, 17, 100250. [CrossRef]

4. Dmitruk, M.; Haratym, W. Morphological differentiation of non-glandular and glandular trichomes on Marrubium vulgare L. Mod. Phytomorphol. 2014, 6, 85.

5. Aćimović, M.; Jeremić, K.; Salaj, N.; Gavarić, N.; Kiprovski, B.; Sikora, V.; Zeremski, T. Marrubium vulgare L.: A phytochemical and pharmacological overview. Molecules 2020, 25, 2898. [CrossRef]

6. Lodhi, S.; Vadnere, G.P.; Sharma, V.K.; Usman, M.R. Marrubium vulgare L.: A review on phytochemical and pharmacological aspects. J. Intercult. Ethnopharmacol. 2017, 6, 429-452. [CrossRef]

7. Mahmoud, A.A.; Gendy, A.S.H.; Said-Al Ahl, H.A.H.; Grulova, D.; Astatkie, T.; Abdelrazik, T.M. Impact of harvest time and water stress on the growth and essential oil components of horehound (Marrubium vulgare). Sci. Hortic. 2018, 232, 139-144. [CrossRef]

8. Said-Al Ahl, H.A.H.; Gendy, A.S.H.; Mahmoud, A.A.; Mohamed, H.F.Y. Essential oil composition of Marrubium vulgare L. cultivated in Egypt. Int. J. Plant. Sci. Ecol. 2015, 1, 138-141.

9. El-Leithy, A.S.; El-Hanafy, S.H.; Omer, E.A.; El-Sayed, A.A.A. Effect of nitrogen and potassium biofertilization on growth, yield and essential oil production of the white horehound, Marrubium vulgare L. plant. J. Hortic. Sci. Ornam. Plants 2013, 5, 46-59.

10. Weel, K.G.C.; Venskutonis, P.R.; Pukalskas, A.; Gruzdiene, D.; Linssen, J.P.H. Antioxidant activity of horehound (Marrubium vulgare L.) grown in Lithuania. Lipid 1999, 101, 395-400. [CrossRef]

11. Morteza-Semnani, K.; Saeedi, M.; Babanezhad, E. The essential oil composition of Marrubium vulgare L. from Iran. J. Essent Oil Res. 2008, 20, 488-489. [CrossRef]

12. Golparvar, A.R.; Hadipanah, A.; Mehrabi, A.M.; Armin, A. Essential oil composition of Marrubium vulgare L. from Iran. Flavour Herb. Drug 2015, 6, 1-5.

13. Zarai, Z.; Kadri, A.; Chobba, I.B.; Mansour, R.B.; Bekir, A.; Mejdoub, H.; Gharsallah, N. The in-vitro evaluation of antibacterial, antifungal and cytotoxic properties of Marrubium vulgare L. essential oil grown in Tunisia. Lipids Hum. Health Dis. 2011, 10, 161. [CrossRef]

14. Kadri, A.; Zarai, Z.; Bekir, A.; Gharsallah, N.; Damak, M.; Gdoura, R. Chemical composition and antioxidant activity of Marrubium vulgare L. essential oil from Tunisia. Afr. J. Biotechnol. 2011, 10, 3908-3914.

15. Abadi, A.; Hassani, A. Essential oil composition and antioxidant activity of Marrubium vulgare L. growing wild in Eastern Algeria. Int. Lett. Chem. Phys. Astron. 2013, 9, 17-24. [CrossRef]

16. Bayir, B.; Gunduz, H.; Usta, T.; Sahin, E.; Ozdemir, Z.; Kayir, O.; Sen, O.; Aksit, H.; Elmastas, M.; Erenler, R. Chemical composition of essential oil from Marrubium vulgare L. leaves. J. New Results Sci. 2014, 6, 44-50.

17. El-Hallous, E.I.; Alsanie, W.F.; Ismail, I.A.; Dessoky, E.S. Utilization of Marrubium vulgare extract as a therapeutic to hepatic damage induced by Carbon Tetrachloride in rats. Int J. Pharm. Res. Allied Sci. 2018, 7, 168-178.

18. Yabrir, B. Chemical composition and biological activities of some Marrubium species essential oil: A review. Chem. J. Mold. 2018, 13, 8-23. [CrossRef]

19. Salehi, N.; Kharazian, N.; Shiran, B. Genetic diversity of Marrubium species from Zagros Region (Iran), using inter simple sequence repeat molecular marker. J. Sci. Islamic Repub. Iran. 2018, 29, 7-19.

20. Kharazian, N.; Hashemi, M. Chemotaxonomy and morphological studies in five Marrubium L. species in Iran. Iran. J. Sci. Technol. Trans. Sci. 2017, 41, 17-31. [CrossRef]

21. Akgul, G.; Ketenoglu, O.; Pinar, N.M.; Kurt, L. Pollen and seed morphology of the genus Marrubium (Lamiaceae) in Turkey. Ann. Bot. Fenn. 2008, 45, 1-10. [CrossRef]

22. Ahvazi, M.; Jamzad, Z.; Balali, G.R.; Saeidi, H. Trichome micro-morphology in Marrubium L. (Lamiaceae) in Iran and the role of environmental factors on their variation. Iran. J. Bot. 2016, 22, 39-58. [CrossRef]

23. Ahvazi, M.; Balali, G.R.; Jamzad, Z.; Saeidi, H. A taxonomical, morphological and pharmacological review of Marrubium vulgare L., an old medicinal plant in Iran. J. Med. Plants 2018, 17, 7-24.

24. Martin, E.; Cetin, O.; Akgul, G.; Hilal, A.Y. New chromosome numbers in the genus Marrubium (horehound) from Turkey. Biol. Divers. Conserv. 2011, 4, 185-188.

25. Buyukkartal, H.N.; Colgecen, H.; Akgul, G. Comparative leaf, stem and root anatomies of taxa Marrubium bourgaei and Marrubium heterodon (Lamiaceae). Aust. J. Crop Sci. 2016, 10, 1516-1522. [CrossRef]

26. Marzouk, R.I.; El-Darier, S.M.; Nour, I.H.; Kamal, S.A. Numerical taxonomic study of Marrubium L. (Lamiaceae) in Egypt. Catrina $2015,13,25-35$. 
27. Sgarbossa, J.; Schmidt, D.; Schwerz, F.; Schwerz, L.; Prochnow, D.; Caron, B.O. Effect of season and irrigation on the chemical composition of Aloysia triphylla essential oil. Rev. Ceres 2019, 66, 85-93. [CrossRef]

28. Castelo, A.V.M.; Del Menezzi, C.H.S.; Resck, I.S. Seasonal variation in the yield and the chemical composition of essential oils from two Brazilian native arbustive species. J. Appl. Sci. 2012, 12, 753-760. [CrossRef]

29. Yavari, A.; Nazeri, V.; Sefidkon, F.; Hassani, M.E. Influence of some environmental factors on the essential oil variability of Thymus migricus. Nat. Prod. Comm 2010, 5, 943-948. [CrossRef]

30. Zouari-Bouassida, K.; Trigui, M.; Makni, S.; Jlaiel, L.; Tounsi, S. Seasonal variation in essential oils composition and the biological and pharmaceutical protective effects of Mentha longifolia leaves grown in Tunisia. Biomed. Res. Int. 2018, 7856517. [CrossRef]

31. Gosztola, B.; Sarosi, S.; Nemeth, E. Variability of the essential oil content and composition of chamomile (Matricaria recutita) affected by weather conditions. Nat. Prod. Commun. 2010, 5, 465-470. [CrossRef]

32. Acimovic, M.; Cvetkovic, M.; Stankovic, J. Effect of weather conditions, location and fertilization on coriander fruit essential oil quality. Essent Oil Bear Plants 2016, 19, 1208-1215.

33. Aziz, E.E.; Badawy, E.M.; Zheljazkov, V.D.; Nicola, S.M.; Fouad, H. Yield and chemical composition of essential oil of Achillea millefolium L. as affected by harvest time. Egypt J. Chem. 2019, 62, 533-540. [CrossRef]

34. Hamdaoui, B.; Wannes, W.A.; Marrakchi, M.; Brahim, N.B.; Marzouk, B. Essential oil composition of two Tunisian horehound species: Marrubium vulgare L. and Marrubium aschersonii Magnus. J. Essent Oil Bear Plants 2013, 16, 608-612. [CrossRef]

35. Laouer, H.; Yabrir, B.; Djeridane, A.; Yousfi, M.; Beldovini, N.; Lamamra, M. Composition, antioxidant and antimicrobial activities of the essential oil of Marrubium deserti. Nat. Prod. Commun. 2009, 4, 1133-1138. [CrossRef] [PubMed]

36. Nagy, M.; Svajdlenka, E. Comparison of essential oils from Marrubium vulgare L. and M. peregrinum L. J. Essent Oil Res. 1998, 10, 585-587. [CrossRef]

37. Kırımer, N.; Kurkcuoglu, M.; Akgul, G.; Baser, K.H.H.; Mahmoud, A.A. Composition of the essential oil of Marrubium anisodon C. Koch of Turkish origin. Rec Nat. Prod. 2015, 9, 234-236.

38. Teimori, M.; Khavari-Nejad, R.A.; Yassa, N.; Nejadsatari, T. Analysis of the essential oil of Marrubium crassidens Bioos. and M. astracanicum Jacq. J. Appl. Sci. 2008, 8, 1793-1795. [CrossRef]

39. Hamedeyazdan, S.; Asnaashari, S.; Fathiazad, F. Characterization of non-terpenoids in Marrubium crassidens Bioss. essential oil. Adv. Pharm. Bull. 2013, 3, 429-432.

40. Golmakani, H.; Rabbani Nasab, H.; Sharifan, M.; Kamali, H.; Yadollahi, A. The essential oil composition and antibacterial activity of Marrubium dubanese Murata from North Khorassan Province, Iran. J. Essent Oil Bear Plants 2016, 19, 963-971. [CrossRef]

41. Hamedeyazdan, S.; Zarei, M.; Salem, A.; Asnaashari, S.; Fathiazad, F. Essential oil of two Iranian horehound species: Marrubium propinquum and Marrubium parviflorum. Pharm. Sci. 2017, 23, 143-149. [CrossRef]

42. Bal, Y.; Kaban, S.; Kirimer, N.; Baser, K.H.C. Composition of the essential oil of Marrubium parviflorum Fisch. et Mey. subsp. oligodon (Boiss.) Seybold. J. Essent Oil Res. 1999, 11, 300-302. [CrossRef]

43. Kaurinovic, B.; Vlaisavljevic, S.; Popovic, M.; Vastag, D.; Djurendic-Brensel, M. Antioxidant properties of Marrubium peregrinum L. (Lamiaceae) essential oil. Molecules 2010, 15, 5943-5955. [CrossRef]

44. Lazari, D.; Skaltsa, H.; Constantinidis, T. Essential oils of Marrubium velutinum Sm. and Marrubium peregrinum L., growing wild in Greece. Flavour Fragr. J. 1999, 14, 290-292. [CrossRef]

45. Hamedeyazdan, S.; Fathiazad, F.; Asnaashari, S. Chemical composition of the essential oil from Marrubium persicum C.A. Mey. (Lamiaceae). Pharm. Sci. 2013, 19, 35-38.

46. Nik, B.Z.; Mirza, M. Composition of the essential oil of Marrubium astracanicum Jacq. J. Essent Oil Res. 2003, 15, 342-343. [CrossRef]

47. Khanavi, M.; Ghasemian, L.; Motlagh, E.H.; Hadjikhoondi, A.; Shafiee, A. Chemical composition of the essential oils of Marrubium parviflorum Fisch. \& C.A. Mey. and Marrubium vulgare L. from Iran. Flavour Fragr. J. 2005, 20, 324-326.

48. Sarikurkcu, C.; Ozer, M.S.; Calli, N.; Popović-Djorđević, J. Essential oil composition and antioxidant activity of endemic Marrubium parviflorum subsp. oligodon. Ind. Crops Prod. 2018, 119, 209-213. [CrossRef]

49. Miloudi, K.; Hamimed, A.; Benmimoun, Y.; Bellebna, Y.; Taibi, A.; Tilmatine, A. Intensification of essential oil extraction of the Marrubium vulgare using pulsed electric field. J. Essent Oil Bear Plants 2018, 21, 811-824. [CrossRef]

50. Zawislak, G. Comparison of chemical composition of the essential oil from Marrubium vulgare L. and M. incanum Desr. during the second year of cultivation. Acta Agrobot. 2015, 68, 59-62. [CrossRef]

51. Petrovic, S.; Pavlovic, M.; Maksimovic, Z.; Milenkovic, M.; Couladis, M.; Tzakou, O.; Niketic, M. Composition and antimicrobial activity of Marrubium incanum Desr. (Lamiaceae) essential oil. Nat. Prod. Commun. 2009, 4, 431-434. [CrossRef] [PubMed]

52. Chemsa, A.E.; Zellagui, A.; Ozturk, M.; Erol, E.; Ceylan, O.; Duru, M.E.; Gherraf, N. Antibiofilm formation, antioxidant and anticholinesterase activities of essential oil and methanol extract of Marrubium deserti de Noe. J. Mater. Env. Sci. 2016, 7, 993-1000.

53. Mohammadhosseini, M. First report of screening of the profiles of the essential oils and volatiles from the aerial parts of Marrubium persicum using classical and advanced methods prior to Gas Chromatographic Mass Spectrometric determination. J. Med. Plants Prod. 2016, 2, 169-180.

54. Agryropoulou, C.; Skaltsa, H. Identification of essential oil components of Marrubium thessalum Boiss. \& Heldr., growing wild in Greece. Nat. Prod. Res. 2012, 26, 1-7.

55. Aćimović, M.; Pezo, L.; Tešević, V.; Čabarkapa, I.; Todosijević, M. QSRR Model for predicting retention indices of Satureja kitaibelii Wierzb. Ex Heuff. essential oil composition. Ind. Crops Prod. 2020, 154, 112752. [CrossRef] 
56. Nekoei, M.; Salimi, M.; Dolatabadi, M.; Mohammadhosseini, M. Prediction of antileukemia activity of berbamine derivatives by genetic algorithm-multiple linear regression. Mon. Chem. 2011, 142, 943. [CrossRef]

57. Nekoei, M.; Mohammadhosseini, M.; Pourbasheer, E. QSAR study of VEGFR-2 inhibitors by using genetic algorithm-multiple linear regressions (GA-MLR) and genetic algorithm-support vector machine (GA-SVM): A comparative approach. Med. Chem. Res. 2015, 24, 3037-3046. [CrossRef]

58. Azar, P.A.; Nekoei, M.; Riahi, S.; Ganjali, M.R.; Zare, K. A quantitative structure-retention relationship for the prediction of retention indices of the essential oils of Ammoides atlantica. J. Serb. Chem Soc. 2011, 76, 891-902. [CrossRef]

59. Todeschini, R.; Consonni, V. Handbook of Molecular Descriptors, Methods and Principles in Medicinal Chemistry; Wiley-VCH Verlag GmbH: Weinheim, Germany, 2000.

60. Bakić, V.V.; Pezo, M.L.; Jovanović, M.P.; Turanjanin, V.M.; Vučićević, B.S.; Mirkov, N.S. Technical analysis of photovoltaic/wind systems with hydrogen storage. Science 2012, 16, 865-875.

61. Arsenović, M.; Pezo, L.; Stanković, S.; Radojević, Z. Factor space differentiation of brick clays according to mineral content: Prediction of final brick product quality. Appl. Clay. Sci. 2015, 115, 108-114. [CrossRef]

62. Boulila, A.; Sanaa, A.; Salem, I.B.; Rokbeni, N.; Mrabet, Y.; Hosni, K.; Fernandez, X. Antioxidant properties and phenolic variation in wild populations of Marrubium vulgare L. (Lamiaceae). Ind. Crops Prod. 2015, 76, 616-622. [CrossRef]

63. Mahdavi, A.; Moradi, P.; Mastinu, A. Variation in terpene profiles of Thymus vulgaris in water deficit stress response. Molecules 2020, 25, 1091. [CrossRef] [PubMed]

64. Noorizadeh, H.; Farmany, A.; Noorizadeh, M. Quantitative structure-retention relationships analysis of retention index of essential oils. Química Nova 2011, 34, 242-249. [CrossRef]

65. Driouche, Y.; Messadi, D. Quantitative structure-retention relationship model for predicting retention indices of constituents of essential oils of Thymus vulgaris (Lamiaceae) (Short communication). J. Serb. Chem Soc. 2019, 84, 405-416. [CrossRef]

66. Acimovic, M.; Pezo, L.; Stankovic Jeremic, J.; Cvetkovic, M.; Rat, M.; Cabarkapa, I.; Tesevic, V. QSRR model for predicting retention indices of geraniol chemotype of Thymus serpyllum essential oil. J. Essent Oil Bear Plants 2020, 23, 464-473. [CrossRef]

67. Pavlić, B.; Teslić, N.; Kojić, P.; Pezo, L. Prediction of the GC-MS retention time for terpenoids detected in sage (Salvia officinalis L.) essential oil using QSRR approach. J. Serb. Chem. Soc. 2020, 85, 9-23. [CrossRef]

68. Noorizadeh, H. Linear and nonlinear quantitative structure linear retention indices relationship models for essential oils. Eurasian J. Anal. Chem 2013, 8, 50-63.

69. Héberger, K. Quantitative structure-(chromatographic) retention relationships. J. Chromatogr A 2007, 1158, 273-305. [CrossRef]

70. Kaliszan, R.; Bączek, T.; Buciński, A.; Buszewski, B.; Sztupecka, M. Prediction of gradient retention from the linear solvent strength (LSS) model, quantitative structure-retention relationships (QSRR), and artificial neural networks (ANN). J. Sep. Sci 2003, 26, 271-282. [CrossRef]

71. Khodadoust, S.; Ghaedi, M.; Hadjmohammadi, M.R. Dispersive nano solid material-ultrasound assisted microextraction as a novel method for extraction and determination of bendiocarb and promecarb: Response surface methodology. Talanta 2013, 116, 637-646. [CrossRef]

72. Wolfender, J.L.; Martia, G.; Thomas, A.; Bertranda, S. Current approaches and challenges for the metabolite profiling of complex natural extracts. J. Chromatogr. A 2015, 1382, 136-164. [CrossRef] [PubMed]

73. Zisi, C.; Sampsonidis, I.; Fasoula, S.; Papachristos, K.; Witting, M.; Gika, H.G.; Nikitas, P.; Pappa-Louisi, A. QSRR modeling for metabolite standards analyzed by two different chromatographic columns using multiple linear regression. Metabolites $2017,7,7$. [CrossRef]

74. Deconinck, E.; Zhang, M.H.; Petitet, F.; Dubus, E.; Ijjaali, I.; Coomans, D.; Vander Heydena, Y. Boosted regression trees, multivariate adaptive regression splines and their two-step combinations with multiple linear regression or partial least squares to predict blood-brain barrier passage: A case study. Anal. Chim. Acta 2008, 609, 13-23. [CrossRef]

75. Yap, C.W. PaDEL-descriptor: An open source software to calculate molecular descriptors and fingerprints. J. Comput. Chem. 2011, 32, 1446-1474. [CrossRef]

76. Goldberg, D.E. Genetic Algorithms in Search, Optimisation and Machine Learning; Addison-Wesley: Boston, MA, USA, 1989.

77. Gramatica, P. Principles of QSAR models validation: Internal and external. Qsar Comb. Sci 2007, 26, 694-701. [CrossRef]

78. Statistica 10 Software (StatSoft, Inc. STATISTICA, ver. 10, Data Analysis Software System). Available online: https://www. statsoft.de/en/software/tibco-statisticatm (accessed on 15 December 2018).

79. Foroughia, M.; Hossein, M.; Azqhandic, A.; Kakhki, S. Bio-inspired, high, and fast adsorption of tetracycline from aqueous media using Fe3O4-g-CN@PEI- $\beta$-CD nanocomposite: Modeling by response surface methodology (RSM), boosted regression tree (BRT), and general regression neural network (GRNN). J. Hazard. Mater. 2020, 388, 121769. [CrossRef]

80. Cheong, Y.L.; Leitão, P.J.; Lakes, T. Assessment of land use factors associated with dengue cases in Malaysia using Boosted Regression Trees. Spat. Spatiotemporal Epidemiol. 2014, 10, 75-84. [CrossRef] [PubMed]

81. Froeschke, J.T.; Froeschke, B.F. Spatio-temporal predictive model based on environmental factors for juvenile spotted seatrout in Texas estuaries using boosted regression trees. Fish. Res. 2011, 111, 131-138. [CrossRef]

82. Sut, N.; Simsek, O. Comparison of regression tree data mining methods for prediction of mortality in head injury. Expert Syst. Appl. 2011, 38, 15534-15539. [CrossRef] 
83. Mazaheri, H.; Ghaedi, M.; Azqhandi, M.A.; Asfaram, A. Application of machine/ statistical learning, artificial intelligence and statistical experimental design for the modeling and optimization of methylene blue and Cd (II) removal from a binary aqueous solution by natural walnut carbon. Phys. Chem. Chem. Phys. 2017, 19, 11299-11317. [CrossRef]

84. Salonen, J.S.; Luoto, M.; Alenius, T.; Heikkilä, M.; Seppä, H.; Telford, R.J.; Birks, H.J. Reconstructing palaeoclimatic variables from fossil pollen using boosted regression trees: Comparison and synthesis with other quantitative reconstruction methods. Quat Sci. Rev. 2014, 88, 69-81. [CrossRef] 